\title{
Reduction of Motion Sickness Through Targeting Histamine $N$-Methyltransferase in the Dorsal Vagal Complex of the Brain
}

\author{
Miao-Miao Chen, ${ }^{1}$ Li-Hua Xu, ${ }^{1}$ Li Chang, ${ }^{1}$ Peng Yin, and Zheng-Lin Jiang \\ Department of Neurophysiology and Neuropharmacology, Institute of Nautical Medicine and Co-innovation Center of \\ Neuroregeneration, Nantong University, Chongchuan District, Nantong, Jiangsu, People's Republic of China
}

Received August 3, 2017; accepted December 20, 2017

\section{ABSTRACT}

To investigate the role of histamine $N$-methyltransferase (HNMT) activity in the development of motion sickness (MS) in the dorsal vagal complex (DVC) to inform the development of new drugs for MS, Beagle dogs and Sprague-Dawley rats were rotated to simulate MS. HNMT expression in the brain stem and DVC was measured. The effects of systemic application of tacrine, an HNMT inhibitor, on the development of MS were observed. Moreover, we microinjected a histamine receptor $\mathrm{H} 1$ inhibitor, promethazine, into the DVC to verify the involvement of histaminergic neurotransmission in MS. Finally, lentiviral vectors were microinjected into the DVC to determine the effects of altered HNMT expression on MS. We found the following: 1) HNMT expression in the medulla oblongata of dogs and rats insusceptible to MS was higher than in susceptible animals; 2) tacrine dose-dependently promoted MS in both animals and raised histamine level in rat medulla oblongata; 3) blocking histaminergic neurotransmission in the DVC with promethazine inhibited MS; 4) rotatory stimulus induced an elevation in HNMT expression, and vestibular training elevated the basal level of HNMT in the DVC during habituation to MS; 5 ) in vivo transfection of a lentiviral vector packaged with the HNMT gene increased HNMT expression in the DVC and reduced MS; and 6) microinjection of a lentiviral vector driving the interference of HNMT gene expression in vivo significantly inhibited HNMT expression in the DVC and exacerbated MS. In conclusion, HNMT expression in the brain stem is inversely correlated with MS development. Increasing HNMT expression or stimulating its activity in the DVC could inhibit MS.

\section{Introduction}

Motion sickness develops after an abnormal vestibular and/or visual stimulation during real or apparent motion and is accompanied by drowsiness, dizziness, cold sweats, pallor, stomach discomfort, nausea, vomiting, and other symptoms of autonomic nervous system distress (Golding and Gresty, 2005; Golding, 2006; Muth, 2006). Motion sickness affects many people traveling or working in special environments, such as sailing, aviation, and spaceflight. Effective medications for the prevention of motion sickness include anticholinergics, antihistamines, sympathomimetic drugs, calcium antagonists, and medicinal herbs (Klöcker et al., 2001; Golding and Gresty, 2005; Murdin et al., 2011). Among the anti-motion sickness drugs, dimenhydrinate and scopolamine are the most effective and most frequently used, but they can cause drowsiness, blurry vision, and performance

This work was supported by the National Natural Science Foundation of China (Grants 81601639, 81372131, and 81671859) and the Administration of Science and Technology of Jiangsu, People's Republic of China (BK20161282), and was partly funded by the Natural Science Foundation of Nantong University (15Z01) and the Priority Academic Program Development of Jiangsu Higher Education Institutions. No potential conflicts of interest relevant to this article were reported.

${ }^{1}$ M.-M.C., L.-H.X., and L.C. contributed equally to this work.

https://doi.org/10.1124/jpet.117.244475. decrement in most subjects (Klöcker et al., 2001; Golding and Gresty, 2005; Herron, 2010; Murdin et al., 2011). Therefore, it is crucial to develop new drugs that can effectively prevent motion sickness with fewer negative side effects, especially for people working in special environments.

Takeda et al. (1986) reported that histamine levels increased in the hypothalamus and pons-medulla oblongata when rats were rotated about two parallel axes to produce motion sickness, but histamine levels were unchanged in bilaterally labyrinthectomized rats. Moreover, $\alpha$-fluoromethylhistidine, an inhibitor of a histamine-synthesizing enzyme, suppressed motion sickness (Takeda et al., 1986). Similarly, when the rats were exposed to $2 g$ hypergravity in an animal centrifuge device to produce motion sickness, histamine release increased in the hypothalamus (Uno et al., 1997). In contrast, neither increased histamine release nor motion sickness was induced by $2 g$ hypergravity in bilaterally labyrinthectomized rats. Pretreatment with $\alpha$-fluoromethylhistidine decreased both the basal and hypergravity-induced release of histamine from the hypothalamus and suppressed motion sickness induced by hypergravity (Uno et al., 1997). These findings suggest that vestibular stimulation activates the histaminergic neuron system in the brain and induces motion sickness. It is well known that antihistamines are effective in preventing motion sickness (Klöcker et al., 2001; Golding and Gresty,

ABBREVIATIONS: CNS, central nervous system; DVC, dorsal vagal complex; GFP, green fluorescent protein; HNMT, histamine $N$ methyltransferase; HPLC, high-performance liquid chromatography; HRP, horseradish peroxidase; qRT-PCR, quantitative real-time polymerase chain reaction; shRNA, short hairpin RNA; SSS, saccharin sodium solution. 
2005; Herron, 2010; Murdin et al., 2011). Overall, the histaminergic neuron system in the brain may play an important role in the development of motion sickness (Takeda et al., 1986; Uno et al., 1997; Bergquist and Dutia, 2006). Histaminergic modulation of the vestibular nucleus and pons-medulla oblongata (including the vomiting center) may also be involved (Bergquist and Dutia, 2006; Haas et al., 2008).

In the mammalian brain, histaminergic neurons are located exclusively in the tuberomammillary nucleus of the posterior hypothalamus and send their axons all over the central nervous system (CNS) (Haas et al., 2008; Thakkar, 2011). Blocking histamine $\mathrm{H} 1$ receptors with antihistamine drugs induces numerous side effects due to the widespread distribution of histaminergic fibers and $\mathrm{H} 1$ receptors in the CNS (Haas et al., 2008; Thakkar, 2011). After release, histamine is inactivated by methylation almost exclusively by the enzyme, histamine- $N$-methyltransferase (HNMT) in the CNS (Haas et al., 2008; Thakkar, 2011). With our knowledge of the histamine degradation pathway, we designed an alternative method that modulated histamine degradation through altered expression of HNMT in a specific area of the brain stem to see whether we could prevent motion sickness.

\section{Materials and Methods}

Animals and Chemicals. For GeneChip analysis, 16 Beagle dogs consisting of 8 males and 8 females $(7.5-10.5 \mathrm{~kg}$ b.wt.) were purchased from the Teaching and Practicing Farm, Institute of Agriculture, Shanghai Communication University (Shanghai, People's Republic of China). The purchase was qualified and certified by Shanghai Laboratory Animal Management Committee [SCXK(Hu)2007-0004]. For the motion sickness test, another 12 Beagle dogs consisting of 6 males and 6 females $(7.8-10.2 \mathrm{~kg}$ b.wt.) were purchased from Yangzhou Sifang Experimental Animal Technology Co., Ltd. (Yangzhou, People's Republic of China). The purchase was qualified and certified by the Jiangsu Laboratory Animal Management Committee [SCXK(Su)2008-0006]. Sprague-Dawley rats with half males and half females (220-250 g b.wt.) and pregnant dams were obtained from the Experimental Animal Center of Nantong University (Nantong, People's Republic of China). The experimenters were blinded for the grouping and genotype of the animals. All procedures used in this study were used in accordance with our institutional guidelines, which comply with international rules and policies and were approved by the Animal Care and Use Committee of Nantong University.

Common inorganic salts were purchased in the People's Republic of China, and cell culture medium (B27, Dulbecco's minimum essential medium, fetal bovine serum, F12, and neurobasal medium) was purchased from Gibco (Carlsbad, CA). GeneChips (GeneChip Canine Genome 2.0 Array) were purchased from Affymetrix China (Shanghai, People's Republic of China). Tacrine was purchased from Shanghai Hebao Chemical Co., Ltd. (Shanghai, People's Republic of China), dimenhydrinate was purchased from Shanghai Huaihai Pharmaceutical Factory (Shanghai, People's Republic of China), and saccharin sodium was purchased from Tianjin North Food Co., Ltd. (Tianjin, People's Republic of China). Pentobarbital sodium, chloral hydrate, glutamine, promethazine, poly-D-lysine, cytosine- $\beta$-D-arabinofuranoside, histamine dihydrochloride, dansyl chloride, mouse anti-rat and $\operatorname{dog} \beta$-actin antibodies, and other chemicals (except those indicated elsewhere) were purchased from Sigma-Aldrich (St. Louis, MO). Rabbit anti-rat, dog HNMT, goat anti-rabbit IgG $(\mathrm{H}+\mathrm{L})$-horseradish peroxidase (HRP), and goat anti-mouse $\operatorname{IgG}(\mathrm{H}+\mathrm{L})-\mathrm{HRP}$ antibodies were purchased from Bioworld China (Nanjing, People's Republic of China).

Motion Sickness Induction via Rotatory Stimulus. The rotatory stimulator for animal use was produced in reference to that

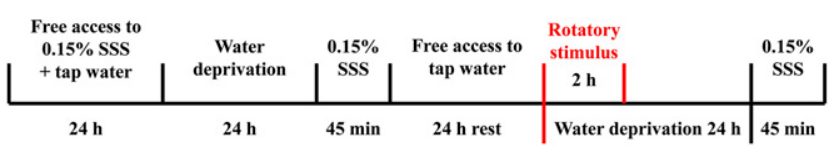

Fig. 1. Protocols of the conditioned taste aversion test in rats.

reported by Crampton and Lucot (1985) and described in our previous study (Li et al., 2005). To induce motion sickness, dogs and rats in the stimulator were rotated in an alternate acceleration and deceleration mode. The acceleration rate was $16 \% \mathrm{~s}^{2}$ for a duration of 7.5 seconds, and a maximal velocity of $120 \% \mathrm{~s}$ was reached. A duration of 2.5 seconds was used for deceleration with a rate of $48 \% \mathrm{~s}^{2}$. Clockwise and counterclockwise rotations were alternately repeated for 30 minutes for the dogs and 120 minutes for the rats.

Vomiting served as the index of motion sickness in the dogs. The same rotatory test was repeated 2 weeks later. After averaging the results of two tests, the dogs with a vomiting onset time of less than 30 minutes were considered susceptible to motion sickness, whereas dogs with a vomiting onset time of more than 30 minutes were considered insusceptible.

Conditioned taste aversion, as suggested by the literature (Ossenkopp, 1983; Fox and McKenna, 1988; Sutton et al., 1988; Gallo et al., 1999; Li et al., 2005), was used as the index of rat motion sickness. Before the rotatory stimulus, saccharin sodium solution (SSS; $0.15 \%$ ) was provided for the rats to drink for 45 minutes when they had been deprived of water for a 24 -hour period and had become familiar with this novel fluid 1 day before water deprivation through free access to $0.15 \%$ SSS in addition to tap water for another 24 hours. After a 24-hour period of rest with free access to tap water, the rats were rotated, and, after another 24-hour period of water deprivation, $0.15 \%$ SSS was then provided for 45 minutes and the SSS intake volume was measured. The same rotatory test was repeated 2 weeks later. After averaging the results of two tests, the rats with a decrease in $0.15 \%$ SSS intake of less than $15 \%$ were considered insusceptible to motion sickness, whereas the rats with a decrease in intake greater than $15 \%$ were considered susceptible. As a control, control rats of both sexes before and after the rotatory stimulus were supplied with tap water instead of $0.15 \%$ SSS for the same duration of 45 minutes after water deprivation for a 24 -hour period. The protocol of the conditioned taste aversion test is shown in Fig. 1.

Adaptive Vestibular Training. For adaptive vestibular training, 14 susceptible rats of both sexes were selected. Seven rats were used as controls without training, and another seven rats were subjected to 1 month of training. The training group was rotated every 2 days with the same rotatory stimulus as described above for a duration of 60 minutes.

Drug Tests on Motion Sickness. Six susceptible dogs were selected for the drug test study, which consisted of a $6 \times 6$ Latin square design with one test conducted every week (i.e., two controls: dimenhydrinate $0.3125 \mathrm{mg} / \mathrm{kg}$; and tacrine $0.1875,0.375$, and $0.75 \mathrm{mg} / \mathrm{kg}$ ) and repeated for 6 weeks, and each dog was subjected to six tests in a random order for the 6 -week experiment design; data from two control dogs in every experiment were averaged as one sample for statistical analysis. The rotatory stimulus was applied for a duration of 30 minutes or until vomiting occurred. Drugs were administered orally 60 minutes before rotatory stimulus, and the control dogs were given an equal volume of $10 \mathrm{ml}$ of normal saline.

Forty-eight insusceptible rats were randomly allocated to four groups (i.e., control, and tacrine $0.675,1.35$, and $2.7 \mathrm{mg} / \mathrm{kg}$ ). Tacrine was delivered via intraperitoneal injection 30 minutes before rotatory stimulus of 2 hours duration, and the control rats received an equal injection volume of $1 \mathrm{ml}$ of normal saline per $200 \mathrm{~g}$ weight.

Intracerebral Microinjection. The rats were anesthetized with $10 \%$ chloral hydrate $(400 \mathrm{mg} / \mathrm{kg}$, i.p.) and placed in a stereotaxic frame (Stoelting, Wood Dale, IL). Over a 15-minute period, $0.5 \mu \mathrm{l}$ of promethazine solution was slowly injected into one side of the dorsal 
TABLE 1

Basal level of HNMT mRNA in the dog brain via GeneChip analysis

\begin{tabular}{lcrrr}
\hline \multicolumn{1}{c}{ Group $(n)$} & Medulla Oblongata & \multicolumn{1}{c}{ Pons } & Diencephalon & Vestibular Cerebellum \\
\hline Susceptible (11) & $1.0 \pm 0.13$ & $1.0 \pm 0.28$ & $1.0 \pm 0.10$ & $1.0 \pm 043$ \\
Insusceptible (5) & $1.68 \pm 0.42$ & $1.18 \pm 0.41$ & $0.89 \pm 0.23$ & $1.88 \pm 0.77$ \\
\hline
\end{tabular}

vagal complex (DVC) using a 1- $\mu$ l microsyringe (Hamilton, Reno, NV) driven by an injector (Quintessential Stereotaxic Injector; Stoelting). The DVC coordinates for the injection site were $14.04 \mathrm{~mm}$ posterior to the bregma, $0.7 \mathrm{~mm}$ lateral to the midline, and a depth of $8.0 \mathrm{~mm}$ under the meninges (Paxinos and Watson, 2005), with a retention time of 10 minutes. The microinjection point was next to the dorsal nucleus of the vagal nerve and the nucleus of the solitary tract. Microinjection for the other side of the DVC was successively conducted in the same way. Control rats were injected with normal saline at an equal volume. Similarly, HNMT-overexpressing and HMNT-inhibitory lentiviruses $(\mathrm{LVs})$ and empty-vector controls $\left(1.5 \times 10^{6}\right.$ transducing units (TU)/2 $\mu \mathrm{l}$ for each side) were respectively injected into both sides of the DVC through a $10-\mu \mathrm{l}$ microsyringe (Hamilton).

Primary Cell Culture. Rat embryos (embryonic days 17-18) were harvested from pregnant rats anesthetized with $10 \%$ chloral hydrate ( $400 \mathrm{mg} / \mathrm{kg}$, i.p.). The embryos were placed in sterile ice-cold Hank's balanced salt solution (Gibco). The embryonic rat brains were taken out, and both sides of the cortex were carefully dissected. The tissue matrix was digested with $0.25 \%$ trypsin (Life Technologies, Carlsbad, $\mathrm{CA})$ at $37^{\circ} \mathrm{C}$ in a shaking water bath for 6 minutes. After mechanical trituration and centrifugation, the final cell pellet was resuspended in Dulbecco's minimum essential medium containing $10 \%$ fetal bovine serum, $10 \% \mathrm{~F} 12$, and $1 \%$ penicillin-streptomycin. The cells were seeded in 24-well poly-D-lysine-coated plates at a density of $5 \times 10^{5}$ cells/ml. Ten hours later, the medium was replaced with neurobasal medium supplemented with $2 \%$ B27, $1 \%$ glutamine, and $1 \%$ penicillinstreptomycin. The cultures were maintained by a $50 \%$ medium substitution every 3 days. The day of plating was counted as in vitro day 0 . On in vitro day 3 , cytosine- $\beta$-D-arabinofuranoside $(5 \mu \mathrm{mol})$ was used to suppress the proliferation of glial cells for 3 days, and then the regular neurobasal medium was recovered. The cell plates were put in a humidified $5 \% \mathrm{CO}_{2}$ incubator at $37^{\circ} \mathrm{C}$.

Nissl Staining and Immunofluorescent Observation. The rats were perfused with $200 \mathrm{ml}$ of saline under $10 \%$ chloral hydrate $(400 \mathrm{mg} / \mathrm{kg}$, i.p.) anesthesia and then with $4 \%$ paraformaldehyde in 0.1 mol phosphate-buffered saline $(\mathrm{pH}$ 7.4). The rat brains were harvested and postfixed for 24 hours. The fixed brains were then protected in phosphate-buffered saline containing 30\% sucrose. For Nissl staining, the brains were coronally cut into slices with a thickness of $30 \mu \mathrm{m}$ using a cryostat (CM1900; Leica, Bensheim, Germany). For immunofluorescence, the brains were cut into slices with a thickness of $5 \mu \mathrm{m}$.

For Nissl staining, the brain slices were mounted with neutral balata, blotted onto slides, and processed through a series of baths in the following order: chloroform (30 minutes), acetone (15 minutes), $100 \%$ ethanol (30 seconds), $95 \%$ ethanol (30 seconds), $70 \%$ ethanol (30 seconds), distilled water (30 seconds, twice), cresyl violet (20 minutes), distilled water (30 seconds, three times), $70 \%$ ethanol ( 1 minutes), 95\% ethanol (1 minutes), 100\% ethanol (1 minutes), chloroform (5 minutes), differentiator (95\% ethanol, added glacial acetic acid until the $\mathrm{pH}$ was $4.1,6$ minutes), $95 \%$ ethanol (2 minutes), $100 \%$ ethanol (3 minutes, twice), xylene ( 2 minutes), and xylene (3 minutes, twice). Afterward, the slices were coverslipped.

For immunofluorescent detection of the HNMT gene transfection, we transfected the cultured cells and the DVC with a lentiviral vector expressing green fluorescent protein (GFP). The brain slices and cultured cells were imaged through a laser confocal microscope (TCS SP8; Leica Microsystems, Wetzlar, Germany) at room temperature and processed with software LAS X version 2.0 (Leica Microsystems).
GeneChip Analysis. The dog brains were harvested under anesthesia (3\% pentobarbital sodium, $1 \mathrm{ml} / \mathrm{kg}$, injected through the small saphenous vein of the foreleg). The medulla oblongata, pons, diencephalon, and vestibular cerebellum were isolated. RNA was prepared by pulverizing tissues in a mortar followed by the addition of TRIzol reagent ( $1 \mathrm{ml} / 100 \mathrm{mg}$ of tissue; Invitrogen, Carlsbad, CA). The RNA quality was evaluated with denaturing gel electrophoresis. RNA was purified with a Qiagen miRNeasy Kit (Qiagen, Düsseldorf, Germany), and RNA concentration was determined using a NanoDrop ND-2000 Spectrophotometer (Thermo Scientific, Waltham, MA).

Each sample (500 ng) of total RNA was used to prepare biotinylated fragmented cDNA according to the Gene-Chip Expression Analysis Technical Manual (Revision 5; Affymetrix, Santa Clara, CA). The Affymetrix GeneChip expression arrays (Canine Genome 2.0 Array) were hybridized in an incubator at $45^{\circ} \mathrm{C}$ at $60 \mathrm{rpm}$ for 16 hours. The arrays were washed and stained using the Fluidics Station 450 (Affymetrix) and scanned using the GeneChip Scanner 7G (Affymetrix). The analysis of gene expression data was accomplished using packages available from the Bioconductor project (www.bioconductor.org). The raw data were normalized using the robust multiarray average background-adjusted, normalized, and log-transformed summarized values to search for differentially expressed genes.

A total of 16 GeneChip arrays were used. Dog brain tissue samples from four susceptible males, seven susceptible females, or four insusceptible males were pooled respectively (i.e., one sample from the medulla oblongata, pons, diencephalon, and vestibular cerebellum was respectively used for final GeneChip array measurement). Another part of the samples from these four brain areas was obtained from one insusceptible female dog. Data from susceptible (11 dogs) and insusceptible (5 dogs) animals of both sexes were compared.

Construction of Recombinant Lentiviral Vector and Transfection to DVC. A recombinant lentiviral vector-expressing rat HNMT-short hairpin RNA (shRNA) (Lv-Hnmt-RNAi) was constructed by Shanghai GeneChem (Shanghai, People's Republic of China) to interfere with the expression of the HNMT gene. HNMT-shRNA was introduced into GV118-RNAi vector that carried the GFP reporter gene driven by the U6 promoter. The following three vectors were designed: LV-shRNA1-HNMT (target sequences: 5'-GGAGACATCATCTGAATAT-3'); LV-shRNA2HNMT (5'-GCTTCCAGGCATAATAGCA-3'); and LV-shRNA3-HNMT (5'-GAGCCAAATGCCGAACAAA-3'). The suppression of HNMT mRNA expression was analyzed by quantitative real-time PCR (qRT-PCR), and HNMT protein levels were quantified using Western blot analysis. The most efficient recombinant vector was used in later experiments. GV118-RNAi vector, which produces a nontargeting sequence, 5'-TTCTCCGAACGTGTCACGT-3', was used as the negative control (LV-shRNA-NC). The recombinant vectors and the shRNA-LVs were cotransfected into human embryonic kidney $293 \mathrm{~T}$ cells and the titer of recombinant lentiviruses was $6 \times 10^{8}$ infectious units $/ \mathrm{ml}$. Another recombinant lentiviral vector based on the GV287-GFP vector was constructed expressing the HNMT gene (Lv-Hnmt; Shanghai GeneChem), and the titer of recombinant lentivirus was $2 \times 10^{8}$ infectious units $/ \mathrm{ml}$.

Lentiviruses of $1.5 \times 10^{6} \mathrm{TU} / 2 \mu \mathrm{l}$ were used for microinjection into each side of the DVC in the in vivo experiment. Ten days after the injection of lentiviruses, the rats were subjected to rotatory stimulus and later measurement of conditioned taste aversion to $0.15 \%$ SSS. In the in vitro transfection experiment, lentiviruses were added to the medium of cultured neurons on in vitro day 7 . After 10 hours, the medium was changed with neurobasal medium supplemented with 

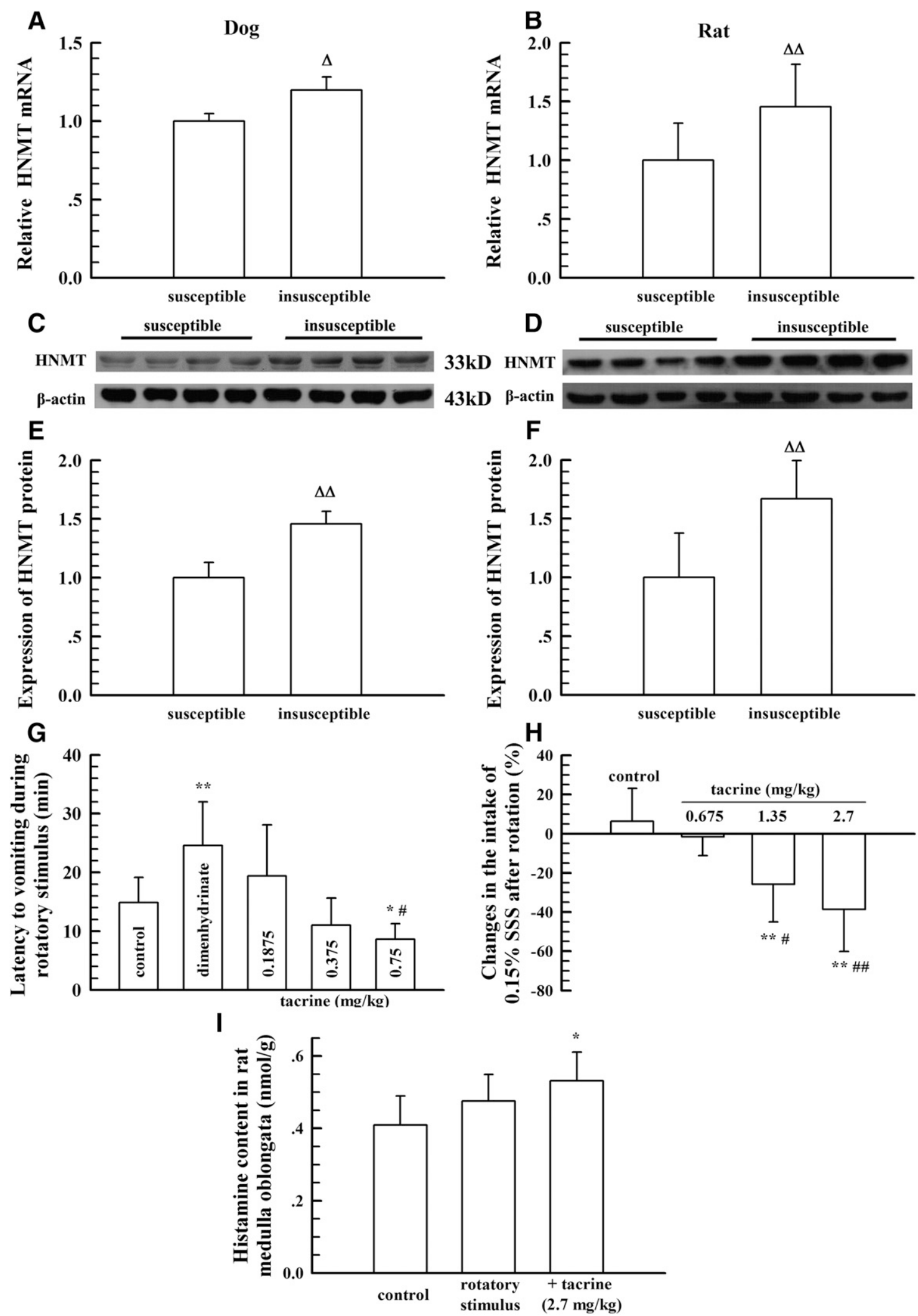

Fig. 2. HNMT activity in the brain influences the development of motion sickness in dogs and rats. (A and B) Basal level of HNMT mRNA, respectively in the medulla oblongata of dogs $(n=4)$ and rats $(n=10)$ susceptible and insusceptible to motion sickness. (C and D) Examples of HNMT protein expression in the medulla oblongatas of dogs and rats, respectively, with different susceptibilities to motion sickness. (E and F) Mean values of HNMT protein expression in the medulla oblongatas of $\operatorname{dogs}(n=4)$ and rats $(n=10)$, respectively, with different susceptibilities to motion sickness. (G) Latency to vomiting after rotatory stimulus in the dogs treated with different doses of tacrine and dimenhydrinate, a positive control drug $(n=6)$. (H) Reduction in $0.15 \%$ SSS intake after rotatory stimulus in the rats treated with different doses of tacrine $(n=12)$. (I) Histamine content in the medulla oblongatas of the rats after rotatory stimulus and tacrine injection $(n=8) . \Delta P<0.05 ; \Delta \Delta P<0.01$, vs. susceptible group; $* P<0.05$; ** $P<0.01$, vs. control; $\# P<0.05$; $\# \# P<0.01$, vs. tacrine $0.1875 \mathrm{mg} / \mathrm{kg}$ in the dogs or $0.675 \mathrm{mg} / \mathrm{kg}$ in the rats. 
A
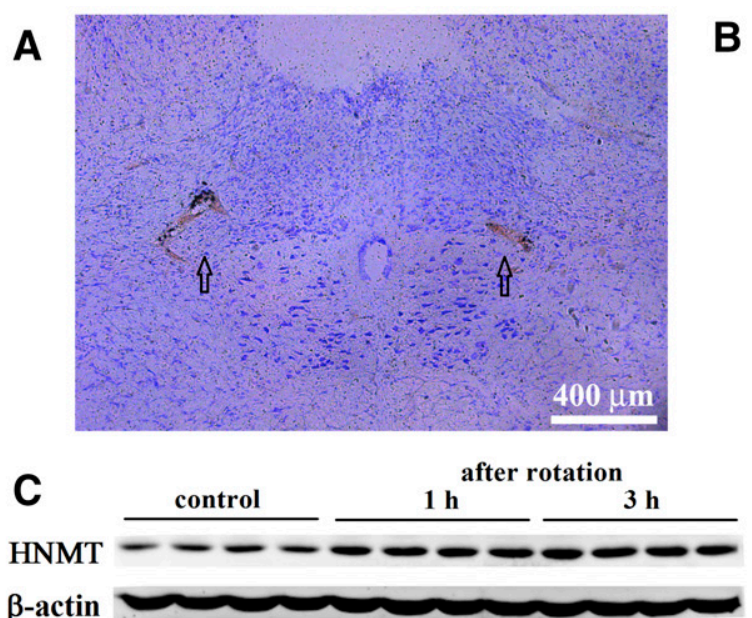

E

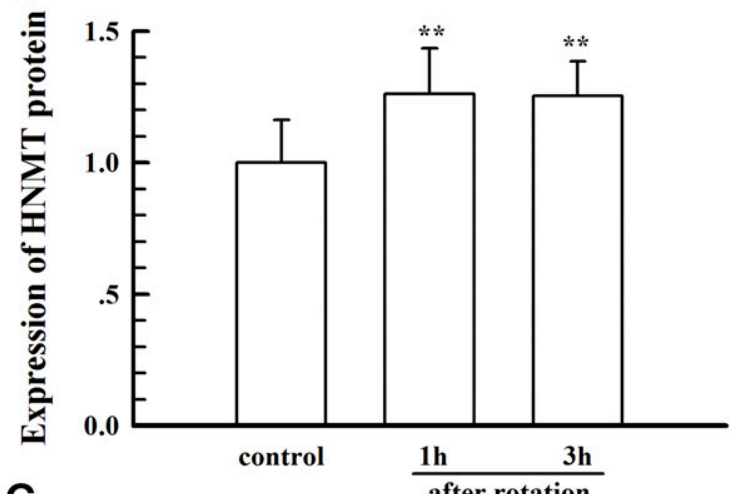

G

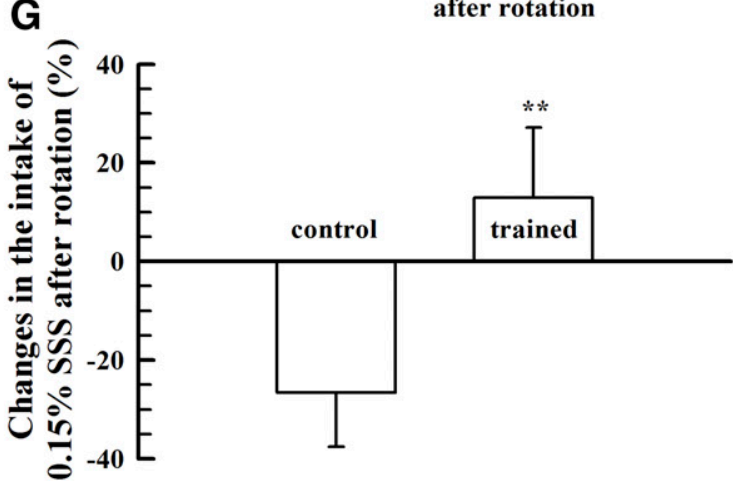

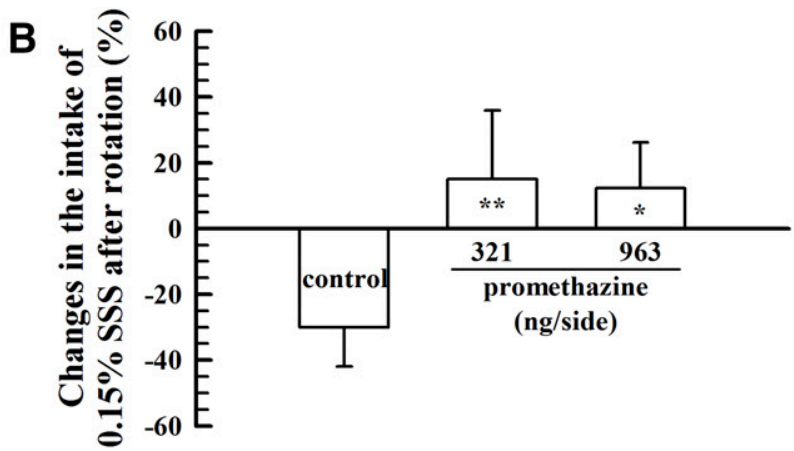

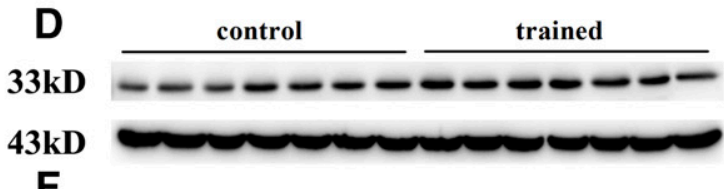

$\mathbf{F}$

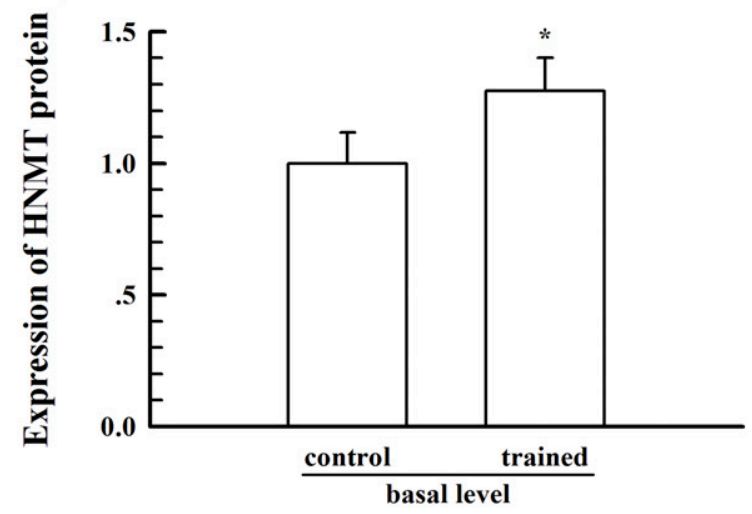

Fig. 3. Microinjection of promethazine into the DVC inhibits motion sickness, whereas rotatory stimulus and vestibular training elevate HNMT levels in the DVC. (A) Indication of the injected DVC areas of both sides (black arrows). (B) Influence of promethazine microinjection into the DVC on the intake volume of $0.15 \%$ SSS after rotatory stimulus $(n=7)$. (C and D) Examples of HNMT expression in the DVC measured with Western blot analysis in the rats after rotatory stimulus and vestibular training, respectively. (E) Mean values of HNMT levels in the DVCs of rats with or without rotatory stimulus $(n=12)$. (F) Mean values of basal HNMT levels in the DVC of rats with or without vestibular training $(n=7)$. (G) Reduction in the intake volume of $0.15 \%$ SSS after rotatory stimulus in rats with or without vestibular training $(n=7) . * P<0.05 ; * * P<0.01$, vs. control.

$2 \%$ B27, $1 \%$ glutamine, and $1 \%$ penicillin-streptomycin. Half of the medium was changed 3 days later. After 4 days, protein was extracted from the cultured neurons for analysis.

Quantitative Real-Time PCR. The rat brains were obtained under $10 \%$ chloral hydrate $(400 \mathrm{mg} / \mathrm{kg}$, i.p.) anesthesia, and the medulla oblongata was isolated on an ice plate. For isolation of the DVC, the rat medulla oblongata was sectioned into $300-\mu \mathrm{m}$-thick slices in ice-cold artificial cerebrospinal fluid using a vibratome (Campden Instruments Ltd., Loughborough, UK). According to the DVC coordinate in the brain atlas (Paxinos and Watson, 2005), the area postrema, nucleus of the solitary tract, and dorsal nucleus of vagus nerve were cautiously isolated for qRT-PCR and Western blot analysis. The dog brains were isolated as described above for GeneChip measurement.
The brain tissues isolated from the rats and dogs or cell cultures were put into TRIzol reagent (Invitrogen) and homogenized with a homogenizer, and the total RNA was then purified. Potential DNA contamination was removed with DNase. One microgram of RNA was used for first-strand cDNA synthesis through retrotranscription according to the manufacturer instructions using oligo(dT) primers and the PrimeScript RT Reagent Kit [Tiangen Biotech (Beijing) Co., Ltd, Beijing, People's Republic of China]. The qRT-PCRs were performed using a Rotor-Gene PCR machine (RG-3000A; Corbett Research Pty. Ltd., Mortlake, NSW, Australia). The qRT-PCR procedures were as follows: a first denaturation step at $95^{\circ} \mathrm{C}$ for 5 seconds, followed by 40 cycles of a $95^{\circ} \mathrm{C}$ denaturation for 5 seconds, $60^{\circ} \mathrm{C}$ annealing for 20 seconds, and $72^{\circ} \mathrm{C}$ extension for 30 seconds. 

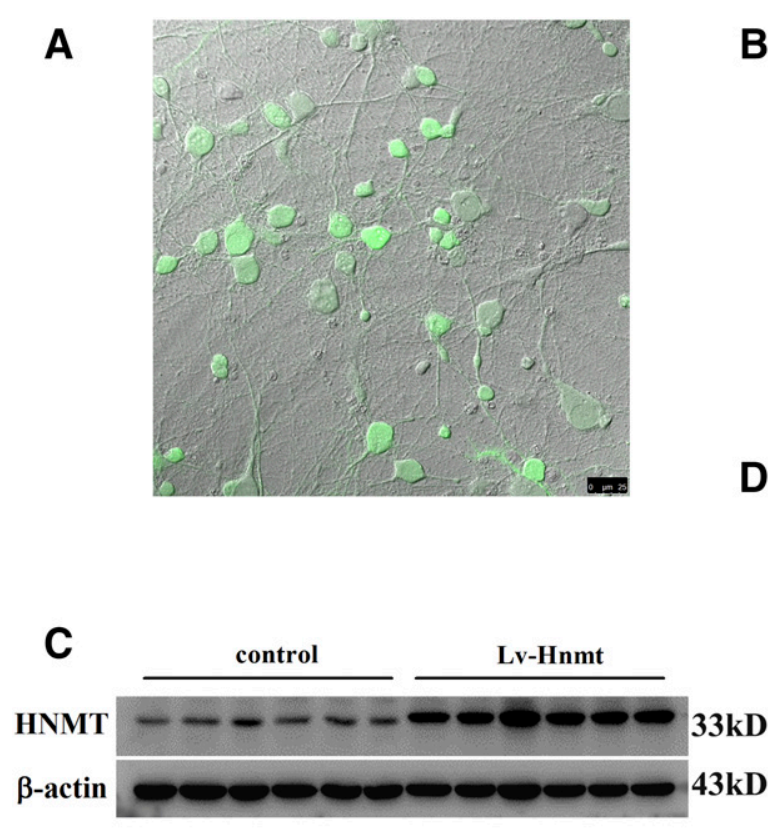
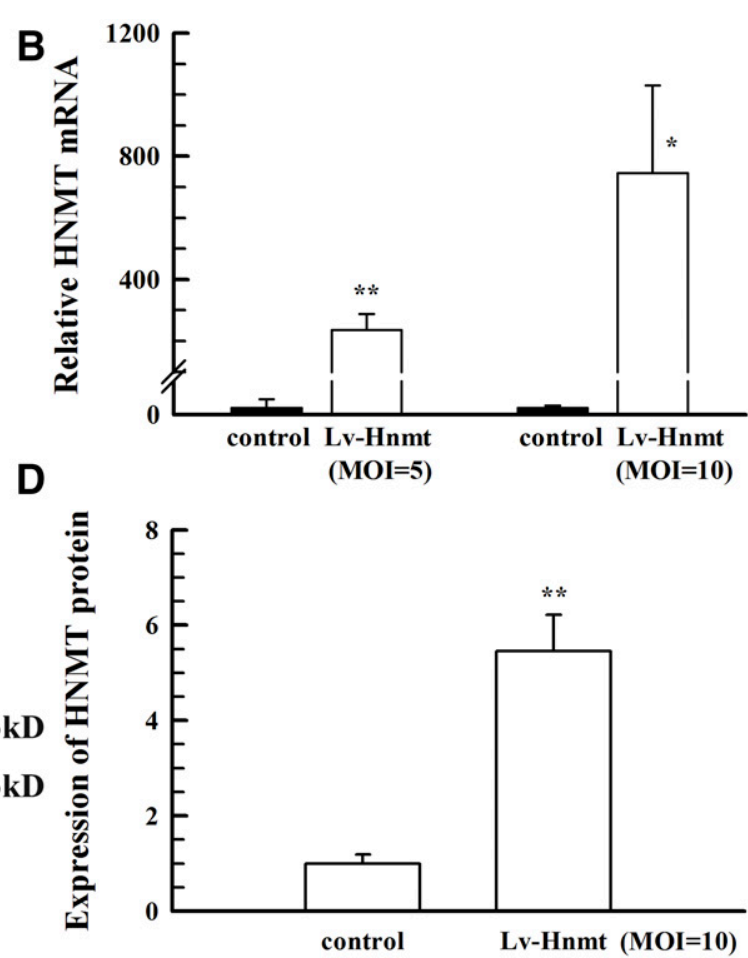

Fig. 4. HNMT overexpression in cultured neurons after transfection of HNMT gene. (A) Merge of a bright-field image of cultured neurons and expression of reporter gene GFP cotransfected with the HNMT gene, indicating successful transfection of HNMT gene into the cultured neurons. Original magnification, $10 \times 40$. Scale bar (black, at the right bottom), $25 \mu \mathrm{m}$. (B) mRNA levels of HNMT after transfection at multiplicity of infection $(\mathrm{MOI})=5$ and $\mathrm{MOI}=10$, respectively $(n=3)$. (C) Examples of HNMT protein expression measured with Western blot analysis after transfection $(\mathrm{MOI}=$ 10). (D) Mean values of HNMT protein expression after transfection $(n=6)$. $* P<0.05$; ** $P<0.01$, vs. negative control.

The amount of cDNA per sample was measured through a SYBR Premix Ex Taq kit (Roche, Basel, Switzerland). The progression of the PCRs was estimated through variations in the SYBR green dye fluorescence attached to double-stranded DNA. All values were normalized to the housekeeping gene $\beta$-actin. The primers used for qRT-PCR of the dog samples were $\beta$-actin (forward 5'-TGGCCGGGACCTGACTGACTACCT-3', reverse 5'-CCCAACCCTGTTAGACCGGCAAGAC-3') and HNMT (forward 5'-CCAGGCATAACAGCAAGGA-3', reverse 5'-TCCCAGCCACTGTTTCCT-3'). The primers used for qRT-PCR of the rat samples were $\beta$-actin (forward 5'-TCTACATGTTCCAGTATGACTC-3', reverse 5'-ACTCCACGACATACTCAGCACC-3') and HNMT (forward 5'-AACAGCACCACTGGACCTCA-3', reverse 5'-TTGCCTCAACCACTATAAAACTCA-3').

Western Blot Analysis. The brain tissues and cell cultures were lysed in lysis buffer containing phenylmethanesulfonyl fluoride (Beyotime, Nantong, People's Republic of China) at $1 \mathrm{mmol}$ and centrifuged at $13,400 \mathrm{~g}$ at $4^{\circ} \mathrm{C}$ for 15 minutes. The supernatant protein content was measured by the bicinchoninic acid method and read on a spectrophotometer. Equal amounts of protein from each sample ( $40 \mu \mathrm{g} /$ lane $)$ were loaded on a $10 \%$ SDS-polyacrylamide gel, electrophoresed, and transferred onto polyvinylidene difluoride membranes (Millipore, Burlington, MA). The membranes were blocked with Tris-buffered saline containing $5 \%$ nonfat milk and $0.1 \%$ Tween-20 at room temperature for 2 hours and incubated at $4^{\circ} \mathrm{C}$ overnight with primary antibodies that were diluted in Tris-buffered saline containing 5\% nonfat milk and 0.1\% Tween-20 to HNMT (1:500) and $\beta$-actin (1:1000). After incubating the blots with goat anti-rabbit IgG $(\mathrm{H}+\mathrm{L})-\mathrm{HRP}$ and goat anti-mouse IgG $(\mathrm{H}+\mathrm{L})-\mathrm{HRP}(1$ : 10,000 ) at room temperature for 2 hours, the immunoreactive bands were visualized using enhanced chemiluminescent agents (Pierce Biotechnology, Waltham, MA) and captured using a Tanon 5200 Multi Chemiluminescent System (Tanon, Shanghai, People's Republic of China). The expression level of each protein was quantified using ImageJ analysis software and normalized to the $\beta$-actin value in the same lane. The same samples were repeatedly analyzed at least two times.
High-Performance Liquid Chromatography Measurement of Histamine. High-performance liquid chromatography (HPLC) measurement of histamine content in the brain tissue was performed with reference to literature (Yamatodani et al., 1985; Altieri et al., 2016). Twenty-four rats were randomly allocated to three groups (i.e., the control, rotation, and tacrine $2.7 \mathrm{mg} / \mathrm{kg}$ groups). Tacrine was delivered via intraperitoneal injection 30 minutes before a 2 -hour rotatory stimulus, and the control rats received an equal volume of normal saline ( $1 \mathrm{ml} / 200 \mathrm{~g}$ weight). Immediately after the rotatory stimulus, the rat brain was harvested by decapitation under anesthesia, and the medulla oblongata was quickly isolated on ice. The brain tissues were weighed and homogenized with a handheld homogenizer after the addition of five volumes of extraction solution $(0.4 \mathrm{~mol}$ perchloric acid), and the supernatants were collected after centrifugation at $12,000 \mathrm{rpm}$ for 20 minutes at $4^{\circ} \mathrm{C}$. All solvents used for HPLC analysis were of HPLC grade. The HPLC system used was the Waters e2695 series (Waters, Milford, MA) equipped with a 2998 Photodiode Array Detector (Waters) and a Sunfire C18 column (5.0 $\mu \mathrm{m}$; column dimension, $6 \times 250 \mathrm{~mm}$; Waters). Each standard solution or sample (100 $\mu \mathrm{l}$ ) was mixed with $20 \mu \mathrm{l}$ of $2 \mathrm{~N}$ sodium hydroxide, $30 \mu \mathrm{l}$ of saturated sodium bicarbonate, and $200 \mu \mathrm{l}$ of dansyl chloride solution $\left(10 \mathrm{mg} / \mathrm{ml}\right.$, prepared in acetone), incubated at $40^{\circ} \mathrm{C}$ for 45 minutes after mixing thoroughly and then cooled down to room temperature in 10 minutes. The residual dansyl chloride was removed with the addition of $10 \mu \mathrm{l}$ of $25 \%$ ammonia solution. Thirty minutes later, the mixture was adjusted to a volume of $1 \mathrm{ml}$ with ammonium acetate $(0.01 \mathrm{~mol}, \mathrm{pH} 7.9)$ and acetonitrile $(1: 1 \mathrm{v} / \mathrm{v})$, and filtered through a syringe filter $(0.45 \mu \mathrm{m}$ pore size). Measurement of histamine level was performed at a flow rate of $1.0 \mathrm{ml} / \mathrm{min}$ with the column temperature at $40^{\circ} \mathrm{C}$. Ammonium acetate was used as solvent $\mathrm{A}$, and acetonitrile as solvent B. The gradient was applied as follows: from $35 \%$ to $70 \%(\mathrm{v} / \mathrm{v})$ solvent B in solvent A for 15 minutes; from $70 \%$ to $95 \%$ (v/v) solvent B in solvent A within 10 minutes; and equilibrated at $95 \%(\mathrm{v} / \mathrm{v})$ solvent B in solvent $A$ for 5 minutes before the next run. The injection volume for 

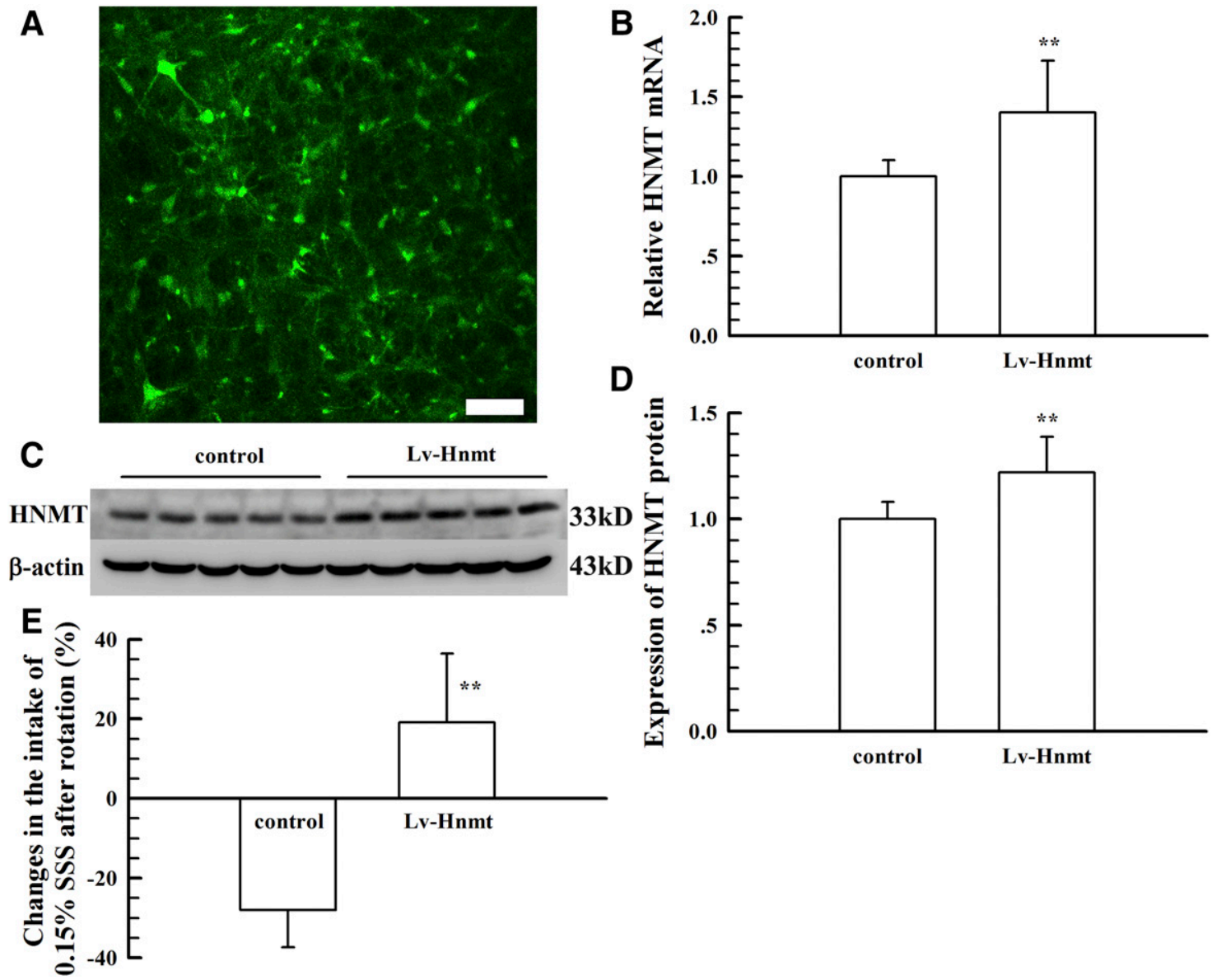

Fig. 5. HNMT overexpression in the DVC of rats transfected with the HNMT gene inhibits motion sickness. (A) Indication of transfected DVC area (green indicates the expression of reporter gene GFP cotransfected with HNMT gene. Original magnification, $10 \times 20$ ). Scale bar (white, at the right bottom), $100 \mu \mathrm{m}$. (B) HNMT mRNA levels after transfection. (C) Examples of HNMT protein expression measured with Western blot analysis after transfection. (D) Mean values of HNMT protein expression after transfection. (E) Intake volume of $0.15 \%$ SSS after rotatory stimulus in the transfected rats relative to negative control rats. For negative control group, $n=10$; for Lv-Hnmt group, $n=12$. $* * P<0.01$, vs. negative control.

standard solutions and samples was $20 \mu \mathrm{l}$, and histamine was detected at a wavelength of $254 \mathrm{~nm}$. The content of tissue histamine was expressed as nanomoles per gram of wet tissue weight (nanomoles per gram) in absolute values.

Statistical Analysis. All data are presented as the mean \pm S.D. The two-group design was analyzed with a Student's $t$ test. The threeor-more group design was analyzed with a one-way analysis of variance followed by the Fisher's least significant difference test for post hoc comparisons between two groups. Differences were considered statistically significant at $P<0.05$.

\section{Results}

Basal Activity of HNMT in the Brain Is Associated with Susceptibility to Motion Sickness in Both Dogs and Rats. Through GeneChip analysis, we found that the basal levels of HNMT mRNA in the dog medulla oblongata and vestibular cerebellum were higher in the insusceptible rats compared with the susceptible rats (Table 1). The role of vestibular cerebellum in the development of motion sickness is still debated (Bard et al., 1947; Wang and Chinn, 1956; Uno et al., 2000; Schmäl, 2013), so we conducted further experiments with qRT-PCR and Western blot analysis to verify the HNMT changes in the medulla oblongata found by GeneChip analysis. As shown in Fig. 2, A and B, basal levels of HNMT
mRNA in both the dog and rat medulla oblongatas were higher in the insusceptible animals than in the susceptible animals $(P<0.05$ or 0.01$)$, which was consistent with the GeneChip analysis. Moreover, the results obtained through Western blot analysis (Fig. 2, C-F) were similar to those measured with GeneChip analysis and qRT-PCR. These results suggest that lower expression of HNMT in the medulla oblongata may be associated with increased susceptibility to motion sickness. Therefore, we hypothesized that the development of motion sickness would be promoted if we inhibited the activity of HNMT. As we expected, tacrine, an inhibitor of HNMT (Cumming et al., 1990; Horton et al., 2005), shortened the latency to vomiting in a dose-dependent manner in the susceptible dogs during rotatory stimulus $(P<0.05)$ (Fig. $2 \mathrm{G})$. Tacrine administration also induced conditioned taste aversion to $0.15 \%$ SSS in a dose-dependent manner in the insusceptible rats after rotatory stimulus $(P<0.01)$ (Fig. $2 \mathrm{H})$. Meanwhile, histamine level in the medulla oblongata was elevated after rotatory stimulus, and tacrine treatment further raised the histamine level in the medulla oblongata of the rats $(P<0.05)$ (Fig. 2I).

Selection of DVC Where HNMT Will Be Targeted for Anti-Motion Sickness. Because the DVC is an important modulator of motion sickness (Yates et al., 1998; Highstein 

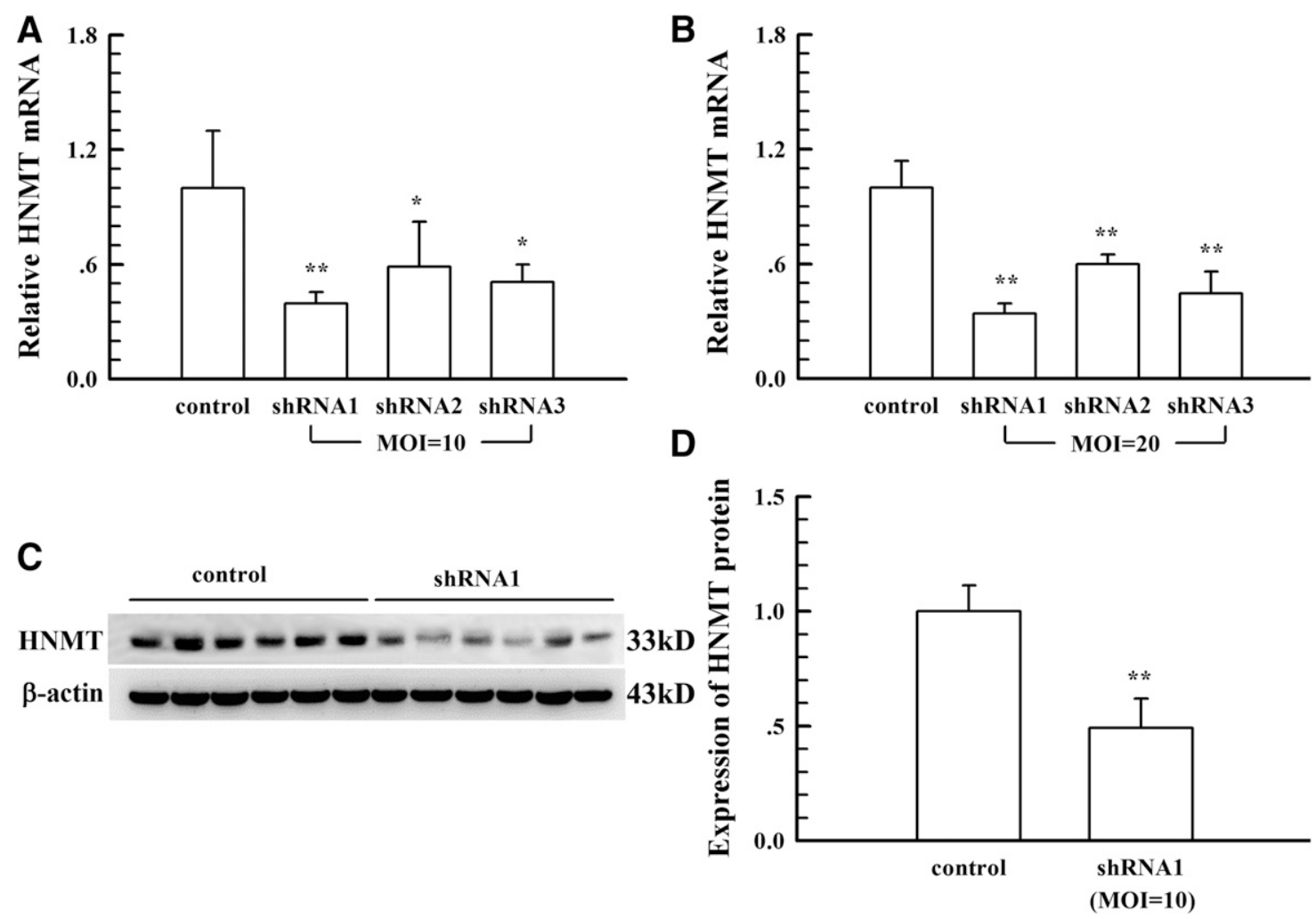

Fig. 6. Downregulation of HNMT expression in cultured neurons after transfection of recombinant lentiviral vectors expressing HNMT-shRNA. (A and B) mRNA levels of HNMT after transfection at multiplicity of infection (MOI) $=10$ and MOI $=20$, respectively $(n=3)$. (C) Examples of HNMT protein expression measured with Western blot analysis after transfection (MOI $=10)$. (D) Mean values of HNMT protein expression after transfection $(n=6)$. $* P<0.05 ; * * P<0.01$, vs. negative control.

and Holstein, 2006; Muth, 2006; Horn, 2008) and receives histaminergic fiber innervations (Panula et al., 1989; Haas et al., 2008), histaminergic neurotransmission in this area (especially the nucleus of solitary tract and dorsal nucleus of vagus nerve) may be involved in the development of motion sickness. Therefore, an anti-histamine drug, promethazine, was used in the present study to block $\mathrm{H} 1$ receptors in the DVC. Microinjection of promethazine into the DVC (Fig. 3A) reduced conditioned taste aversion to $0.15 \%$ SSS induced by rotatory stimulus, and the intake volume of $0.15 \%$ SSS was increased when compared with that in control rats $(P<0.01)$ (Fig. 3B). Subsequently, changes in HNMT expression in the rat DVC after rotatory stimulus were observed. As shown in Fig. 3, C and E, the rotatory stimulus induced an elevation in HNMT expression in the DVC $(P<0.01)$ at 1 and 3 hours after rotation. In addition, basal HNMT level was significantly elevated $(P<0.05)$ (Fig. 3, D and F) after vestibular training through repeated rotatory stimuli was performed for 1 month, whereas the rats habituated to motion sickness (Fig. 3G). These results suggest that motion sickness may be reduced if we inhibit histaminergic neurotransmission using H1 receptor inhibitor promethazine or through elevating HNMT expression in the rat DVC. Therefore, the DVC was selected for further experiments where HNMT was targeted to see its role in the development of motion sickness in the rats.

HNMT Overexpression Induced by Lentiviral Vector Transfection in the DVC Inhibits Motion Sickness. In cultured cortical neurons on in vitro day 7 , lentiviruses expressing the HNMT gene were added to the culture medium. HNMT mRNA and protein levels were significantly increased 4 days after transfection $(P<0.05$ or 0.01$)$ (Fig. 4).
We also transfected the DVC with lentiviruses expressing the HNMT gene in rats using microinjection. Ten days after microinjection, both HNMT mRNA and protein levels were elevated significantly $(P<0.01)$ (Fig. 5, B-D). Meanwhile, the conditioned taste aversion to $0.15 \%$ SSS was reduced in rats subjected to rotatory stimulus when compared with negative control rats $(P<0.01)$ (Fig. 5E).

Interfering with HNMT Gene Expression in the DVC through Lentiviral Vector Transfection Promotes Motion Sickness. To interfere with HNMT gene expression, three recombinant lentiviral vectors expressing rat HNMT-shRNA were constructed as LV-shRNA1-HNMT, LV-shRNA2-HNMT, and LV-shRNA3-HNMT. Interference with HNMT gene expression was first observed in cultured cortical neurons 4 days after transfection. As shown in Fig. 6, $\mathrm{A}$ and $\mathrm{B}$, transfection of all three recombinant lentiviral vectors, LV-shRNA1-HNMT, LV-shRNA2-HNMT, and LV-shRNA3-HNMT, downregulated the expression of HNMT mRNA $(P<0.05$ or $\mathrm{P}<0.01)$. LV-shRNA1-HNMT was the most efficient, thus LV-shRNA1-HNMT was selected for the remaining experiments. Relative to the negative control (LV-shRNA-NC), HNMT protein expression was reduced significantly after transfection of LV-shRNA1-HNMT $(P<0.01)$ (Fig. 6, C and D).

We transfected the rat DVC with LV-shRNA1-HNMT. Ten days after lentivirus injection, both HNMT mRNA and protein levels were significantly downregulated $(P<0.01)$ (Fig. 7, A-C). After the rats were subjected to rotatory stimulus, they consumed less $0.15 \%$ SSS after the transfection of LV-shRNA1-HNMT when compared with rats in the negative control group $(P<0.01)$ (Fig. 7D). 

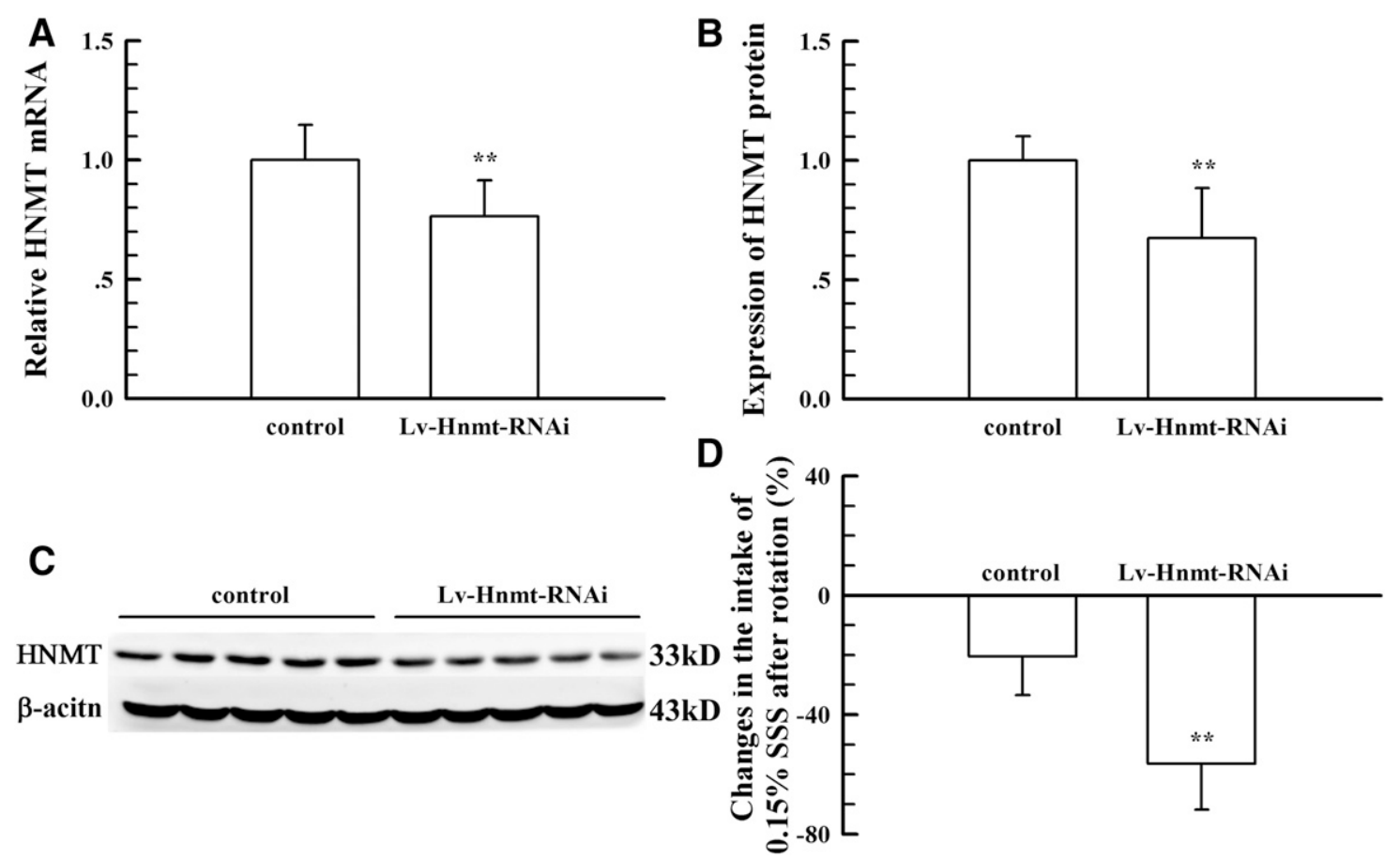

Fig. 7. Downregulation of HNMT expression in the DVC of rats transfected with LV-shRNA1-HNMT promotes motion sickness. (A) HNMT mRNA levels after transfection. (B) Mean values of HNMT protein expression after transfection. (C) Examples of HNMT protein expression measured with Western blot analysis after transfection. (D) Intake volume of $0.15 \%$ SSS after rotatory stimulus in the transfected rats relative to negative control rats. For negative control group, $n=9$; for LV-shRNA1-HNMT group, $n=10$. ${ }^{* *} P<0.01$, vs. negative control.

\section{Discussion}

For the first time, our results revealed that susceptibility to motion sickness is associated with HNMT expression. HNMT mRNA and protein levels in the medulla oblongata were negatively correlated with susceptibility to motion sickness, as higher expression levels resulted in lower susceptibility to motion sickness in dogs and rats. Meanwhile, inhibition of HNMT with tacrine exacerbated motion sickness in both the dog and the rat. These results imply that motion sickness may be inhibited by the promotion of HNMT activity in the brain.

However, there are no known activators of HNMT at present; thus, we could not use HNMT activators to verify these results in reverse. Due to the lack of vomiting reflex, conditioned taste aversion was used as the index of motion sickness in the rats (Ossenkopp, 1983; Fox and McKenna, 1988; Sutton et al., 1988; Gallo et al., 1999; Li et al., 2005); thus, bias or mistake may be made when results obtained in the experiments using rats are used to explain a phenomenon developed in the humans. Therefore, dogs were used in the experiments of the present study to verify the results from rat experiments.

It is well known that histaminergic fibers are distributed all over the CNS (Haas et al., 2008; Thakkar, 2011). The histaminergic neuron system could be activated by vestibular stimulation (Takeda et al., 1986; Uno et al., 1997). Currently, histamine $\mathrm{H} 1$ receptor inhibitors are widely used for the prevention of motion sickness (Klöcker et al., 2001; Golding and Gresty, 2005; Herron, 2010; Murdin et al., 2011; Schmäl, 2013). The vestibular nucleus, medulla oblongata, and especially the DVC are important regions involved in the development of motion sickness (Takeda et al., 1986; Yates et al., 1998; Highstein and Holstein, 2006; Muth, 2006; Horn, 2008;
Schmäl, 2013), and histaminergic innervation exists in both the vestibular nucleus and medulla oblongata (Panula et al., 1989; Steinbusch, 1991; Highstein and Holstein, 2006; Haas et al., 2008; Poole et al., 2008). Histaminergic modulation of the vestibular nucleus and pons-medulla oblongata (including the vomiting center) is potentially involved in motion sickness (Takeda et al., 1986; Bergquist and Dutia, 2006; Haas et al., 2008; Poole et al., 2008; Schmäl, 2013). Moreover, vestibular innervation of the DVC may be an important pathway in the vestibular-autonomic reflex of motion sickness (Highstein and Holstein, 2006; Muth, 2006). Therefore, we designed further experiments to understand the involvement of histaminergic modulation on the DVC in the development of motion sickness. We found that microinjection of the histamine H1 receptor inhibitor promethazine into the DVC of the rats reduced conditioned taste aversion induced by rotatory stimulus. In addition, exposure to rotatory stimulus induced a responsive elevation in HNMT expression levels in the DVC, whereas histamine content in the medulla oblongata of the rats was elevated, and tacrine treatment promoted motion sickness followed by a further increase in histamine content in the medulla oblongata. Moreover, repeated rotatory stimulus exposure habituated rats to motion sickness and elevated HNMT levels in the DVC. Therefore, it is suggested that vestibular stimulation potentially promotes histaminergic neurotransmission, increases histamine release, and stimulates the activity and expression of HNMT in the DVC. Additionally, higher basal levels of HNMT in the DVC could inhibit the overactivity of histaminergic neurotransmission and thereby reduce susceptibility to motion sickness.

Moreover, we conducted further experiments to help us understand the relationship of HNMT with motion sickness. We observed the influence of HNMT on motion sickness by 
modulating HNMT expression in the DVC with gene transfection or RNA interference. Similar to the anti-motion sickness effects of promethazine, a positive control drug we used in the present study, elevation of HNMT gene expression in the DVC inhibited the motion sickness in rats. In contrast, introduction of recombinant lentiviral vectors expressing rat HNMT-shRNA into the DVC to reduce the expression of HNMT promoted motion sickness in rats. These results are consistent with the motion sickness-promoting effects of tacrine mentioned above and suggest that motion sickness could be reduced through targeting histamine $N$-methyltransferase to increase its activity in the dorsal vagal complex of the brain.

Antihistamine drugs are widely administered to prevent motion sickness at present, and the efficacy is remarkable (Klöcker et al., 2001; Golding and Gresty, 2005; Herron, 2010; Murdin et al., 2011; Schmäl, 2013); however, they have many undesirable side effects (Herron, 2010) that are caused by direct block of histamine $\mathrm{H} 1$ receptors with widespread distribution in the brain because histaminergic neurons send their axons to areas almost all around the brain (Haas et al., 2008; Thakkar, 2011). Targeting the histamine degradation pathway as indicated in the present study, activating HNMT or elevating the expression of HNMT will be an alternative method to prevent motion sickness without direct block of the histaminergic neurotransmission in the brain. In the future, we have to conduct many experiments to verify whether this method could avoid many undesirable side effects induced by histamine $\mathrm{H} 1$ receptor blockers or might induce new types of side effects.

In conclusion, our results suggest that lower expression of HNMT in the brain leads to higher susceptibility of individuals to motion sickness but that higher expression of HNMT in the brain contributes to lower susceptibility of individuals to motion sickness; and that inhibiting the activity or reducing the expression of HNMT in the DVC of the brain stem will promote the development of motion sickness, but elevating the expression or stimulating the activity of HNMT could prevent motion sickness. Novel drugs that specifically target HNMT are not currently available and would have to be developed in the future.

\section{Authorship Contributions}

Participated in research design: Chen, $\mathrm{Xu}$, Chang, Yin, and Jiang. Conducted experiments: Chen, $\mathrm{Xu}$, Chang, and Yin.

Performed data analysis: Chen, $\mathrm{Xu}$, Chang, Yin, and Jiang.

Wrote or contributed to the writing of the manuscript: Chen, $\mathrm{Xu}$, Chang, and Jiang.

\section{References}

Altieri I, Semeraro A, Scalise F, Calderari I, and Stacchini P (2016) European official control of food: determination of histamine in fish products by a HPLC-UV-DAD method. Food Chem 211:694-699.

Bard P, Woolsey CN, Snider RS, Mountcastle VB, and Bromiley RB (1947) Delimitation of central nervous mechanisms involved in motion sickness. Fed Proc 6:72. Bergquist F and Dutia MB (2006) Central histaminergic modulation of vestibular function - a review. Sheng Li Xue Bao 58:293-304.
Crampton GH and Lucot JB (1985) A stimulator for laboratory studies of motion sickness in cats. Aviat Space Environ Med 56:462-465.

Cumming P, Reiner PB, and Vincent SR (1990) Inhibition of rat brain histamine-Nmethyltransferase by 9 -amino-1,2,3,4-tetrahydroacridine (THA). Biochem Pharmacol 40:1345-1350.

Fox RA and McKenna S (1988) Conditioned taste aversion induced by motion is prevented by selective vagotomy in the rat. Behav Neural Biol 50:275-284.

Gallo M, Marquez SL, Ballesteros MA, and Maldonado A (1999) Functional blockade of the parabrachial area by tetrodotoxin disrupts the acquisition of conditioned taste aversion induced by motion-sickness in rats. Neurosci Lett 265:57-60.

Golding JF (2006) Motion sickness susceptibility. Auton Neurosci 129:67-76.

Golding JF and Gresty MA (2005) Motion sickness. Curr Opin Neurol 18:29-34

Haas HL, Sergeeva OA, and Selbach O (2008) Histamine in the nervous system. Physiol Rev 88:1183-1241.

Herron DG (2010) The ups and downs of motion sickness. Am J Nurs 110:49-51.

Highstein SM and Holstein GR (2006) The anatomy of the vestibular nuclei. Prog Brain Res 151:157-203.

Horn CC (2008) Why is the neurobiology of nausea and vomiting so important? Appetite 50:430-434.

Horton JR, Sawada K, Nishibori M, and Cheng X (2005) Structural basis for inhibition of histamine N-methyltransferase by diverse drugs. J Mol Biol $\mathbf{3 5 3}$ 334-344.

Klöcker N, Hanschke W, Toussaint S, and Verse T (2001) Scopolamine nasal spray in motion sickness: a randomised, controlled, and crossover study for the comparison of two scopolamine nasal sprays with oral dimenhydrinate and placebo. Eur $J$ Pharm Sci 13:227-232.

Li X, Jiang ZL, Wang GH, and Fan JW (2005) Plasma vasopressin, an etiologic factor of motion sickness in rat and human? Neuroendocrinology 81:351-359.

Murdin L, Golding J, and Bronstein A (2011) Managing motion sickness. BMJ 343: d7430.

Muth ER (2006) Motion and space sickness: intestinal and autonomic correlates. Auton Neurosci 129:58-66.

Ossenkopp KP (1983) Area postrema lesions in rats enhance the magnitude of body rotation-induced conditioned taste aversions. Behav Neural Biol 38:82-96.

Panula P, Pirvola U, Auvinen S, and Airaksinen MS (1989) Histamine-immunoreactive nerve fibers in the rat brain. Neuroscience 28:585-610.

Paxinos G and Watson C (2005) The Rat Brain in Stereotaxic Coordinates-The New Coronal Set, 5th ed, Academic Press, London.

Poole SL, Lewis DI, and Deuchars SA (2008) Histamine depolarizes neurons in the dorsal vagal complex. Neurosci Lett 432:19-24.

Schmäl F (2013) Neuronal mechanisms and the treatment of motion sickness. Pharmacology 91:229-241.

Steinbusch HW (1991) Distribution of histaminergic neurons and fibers in rat brain. Comparison with noradrenergic and serotonergic innervation of the vestibular system. Acta Otolaryngol Suppl 479:12-23.

Sutton RL, Fox RA, and Daunton NG (1988) Role of the area postrema in three putative measures of motion sickness in the rat. Behav Neural Biol 50:133-152.

Takeda N, Morita M, Kubo T, Yamatodani A, Watanabe T, Wada H, and Matsunaga $\mathrm{T}$ (1986) Histaminergic mechanism of motion sickness. Neurochemical and neuropharmacological studies in rats. Acta Otolaryngol 101:416-421.

Thakkar MM (2011) Histamine in the regulation of wakefulness. Sleep Med Rev 15: 65-74.

Uno A, Takeda N, Horii A, Morita M, Yamamoto Y, Yamatodani A, and Kubo T (1997) Histamine release from the hypothalamus induced by gravity change in rats and space motion sickness. Physiol Behav 61:883-887.

Uno A, Takeda N, Kitahara T, Sakata Y, Yamatodani A, and Kubo T (2000) Effects of vestibular cerebellum lesion on motion sickness in rats. Acta Otolaryngol 120: 386-389.

Wang SC and Chinn HI (1956) Experimental motion sickness in dogs; importance of labyrinth and vestibular cerebellum. Am J Physiol 185:617-623.

Yamatodani A, Fukuda H, Wada H, Iwaeda T, and Watanabe T (1985) Highperformance liquid chromatographic determination of plasma and brain histamine without previous purification of biological samples: cation-exchange chromatography coupled with post-column derivatization fluorometry. J Chromatogr A 344: 115-123.

Yates BJ, Miller AD, and Lucot JB (1998) Physiological basis and pharmacology of motion sickness: an update. Brain Res Bull 47:395-406.

Address correspondence to: $\mathrm{Dr}$. Li-Hua $\mathrm{Xu}$, Department of Neurophysiology and Neuropharmacology, Institute of Nautical Medicine, Nantong University, 9 Seyuan Road, Chongchuan District, Nantong, Jiangsu 226019, People's Republic of China. E-mail: shirley19870622@126.com; or Dr. Zheng-Lin Jiang, Department of Neurophysiology and Neuropharmacology, Institute of Nautical Medicine, Nantong University, 9 Seyuan Road, Chongchuan District, Nantong, Jiangsu 226019, People's Republic of China. E-mail: jiangzl@ntu.edu.cn 